\title{
High-throughput sequencing reveals the change of microRNA expression caused by allopolyploidization in Cucumis
}

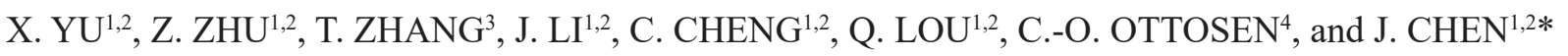 \\ Nanjing Agricultural University, Nanjing,210095 P.R. China ${ }^{1}$ \\ National Key Laboratory of Crop Genetics and Germplasm Enhancement, Nanjing, 210095 P.R. China ${ }^{2}$ \\ Shanghai Qingpu Vegetable Technology Promotion Station, Shanghai, 201799 P.R. China ${ }^{3}$ \\ Department of Food Science, Aarhus University, Aarhus, 8200 Denmark $^{4}$
}

\begin{abstract}
Allopolyploidy is long recognized as an essential driving force in plant evolution. Recent studies have demonstrated that small RNAs, including microRNAs (miRNAs), play important roles in the process of allopolyploidy. However, the question that how the distinct parent-of-origin miRNAs are maintained in allopolyploids and how these small RNAs affect gene expression and phenotype remain to be answered. Therefore, we investigated the miRNA expression profiles of a synthesized allotetraploid, Cucumis $\times$ hytivus and its parents. The different developmental stages of leaves of C. $\times$ hytivus showing contrasting leaf colour were compared as well. Following high-throughput sequencing, 546 conserved d 287 novel miRNAs were identified. The expressions of nine miRNAs obtained by real-time quantitative PCR were consistent with the sequencing results. We detected that 15 miRNAs were divergently expressed between the parent species, and 23 miRNAs were differentially expressed in $C$. $\times$ hytivus compared to either of its parents or both, which suggests the significant effect of allopolyploidization on miRNAs accumulation. Additionally, 26 conserved and 13 novel miRNAs differed in expression between the young and mature leaves of $C$. $\times$ hytivus, indicating an essential role of miRNA-mediated regulation of leaf development following allopolyploidization. This study enriches the context of allopolyploidy effect on miRNAs and lays a foundation for the elucidation of the miRNA-mediated regulatory mechanism in phenotypic variation in allopolyploids.
\end{abstract}

Additional key words: cucumber, gene expression, leaf development, next-generation sequencing.

\section{Introduction}

Polyploidy, including autopolyploidy (within species) and allopolyploidy (between species), is prevalent in angiosperm and plays an important role in the evolutionary force in plants (Jiao et al. 2011). However, the research concerning allopolyploidization is restricted within a few plant species, i.e. wheat (Kenan-Eichler et al. 2011), cotton (Wang and Zhang 2015), Arabidopsis (Ha et al. 2009), Brassica species (Ghani et al. 2014, Shen et al. 2014) and chrysanthemum (Wang et al. 2014). In addition, the existing allotetraploids are often formed between extant progenitors more than million years ago, and the exact progenitors are vanished or evolved (Guan et al. 2014). Synthesized allopolyploids with known parents provide perfect solutions to allow researchers to investigate the exact effects of polyploidization through more critical comparisons of allopolyploids and parents.

The narrow genetic base of cucumber (Cucumis sativus L.) is a significant bottleneck in the development of cucumber cultivars to meet the needs of growers and industry. Through interspecific hybridization between a wild Cucumis species C. hystrix Chakrav. $(2 \mathrm{n}=2 \mathrm{x}=24, \mathrm{HH})$ and cultivated cucumber (C. sativus L. cv. Beijingjietou, $2 \mathrm{n}=2 \mathrm{x}=14, \mathrm{CC})$, and somaclonal variation, $C$. $\times$ hytivus (HHCC) is obtained (Chen and Kirkbride 2000). Previous studies reported that phenotypic differences (e.g., flowering time, fruit shape, and other morphological traits) can be observed between $C . \times$ hytivus and its diploid parents (Chen et al. 2002, 2003). Extensive genomic and epigenetic changes are also detected in $C . \times$ hytivus (Chen et al. 2007, 2008). These results suggest that $C$. $\times$ hytivus, a newly synthesized allotetraploid, is an ideal model system to study the allopolyploidization and clear genetic relationship of hybrid with its diploid parents.

MicroRNAs (miRNAs) are approximately $21-22$ nucleotides (nt) long noncoding RNA molecules that play crucial regulatory roles in animals and plants (Bartel

Submitted 21 March 2019, last revision 26 August, accepted 2 September 2019.

Abbreviation: miRNA - microRNA.

Acknowledgements: This work was supported by the Fundamental Research Funds for the Central Universities (KYZ201735), the Natural Science Foundation of Jiangsu Province, China (BK20180536), and the China Postdoctoral Science Foundation (2017M611834).

* Corresponding author; fax: (+86) 02584396279, e-mail: jfchen@njau.edu.cn 
2004). To date, thousands of miRNAs have been identified across the plant kingdom thanks to the boost in the nextgeneration high-throughput sequencing technology (Morin et al. 2008, Mao et al. 2012, Yang et al. 2013, Cao et al. 2014, Ghani et al. 2014, ). Many miRNAs are conserved among plants species, whereas some are specific to certain plant species or lineages (Zhang et al. 2006, Ha et al. 2008). Moreover, conserved miRNAs do not necessarily display the same expression or pattern in different species or even at different stages within a species (Ha et al. 2008). Therefore, sequence and expression divergence in miRNAs between species may affect miRNA accumulation and target regulation in allopolyploids that contain two or more divergent genomes, leading to developmental changes and/or phenotypic variation in the new species.

This study aims at identifying conserved miRNAs and predicting novel miRNAs in the synthesized allotetraploid and revealing differences of miRNAs both between sub-species in Cucumis and between different leaf developmental stages that may contribute to the variation in phenotypes.

\section{Materials and methods}

Plants and sample collection: Single seeds after soaking and pre-germination were sown in a plug tray and then cultured in climate chambers (RDN-1000E-4, Dongnan Instruments, Ningbo, China) at air temperatures of $25 / 20^{\circ} \mathrm{C}$ (day/night), a 14-h photoperiod, a light intensity of $280 \pm 20 \mu \mathrm{mol} \mathrm{m}^{-2} \mathrm{~s}^{-1}$, and a $60 \pm 10 \%$ relative humidity. On the $20^{\text {th }}$ day after sowing, healthy and uniform seedlings were transferred to plastic plots $(11 \mathrm{~cm}$ diameter, $9 \mathrm{~cm}$ height) and cultured in the climate chambers with the same climate setting. The seedlings were irrigated by flooding the bench for 10 min two times per day with the Japanese Garden test formula. Twenty days later, uniform plants were used for sample collection. The young leaf (first leaf counting from the top) and the mature leaf (the first fully developed leaf) of self-cross plants $\left(\mathrm{S}_{10}\right)$ of synthesized new species, Cucumis $\times$ hytivus (HHCC_Y and HHCC_M) and the mature leaves of the diploid parents, Cucumis hystrix Chakraw. (HH_M) and Cucumis sativus L. (CC_M) were used for sequencing. Leaf samples were separated into two packs, one for sequencing and the other for real-time quantitative PCR (qPCR) analysis.

Small RNA libraries construction for high-throughput sequencing: There were two biological replicates for each leaf samples, which were differed with number 1 and 2 . To construct the eight sRNA libraries, leaf samples were subjected to sRNAs extraction with TruSeq small RNA sample preparation kits (Illumina, San Diego, CA, USA). After isolation and ligation, the sRNAs were reversely transcribed M-MLV reverse transcriptase (Invitrogen, Carlsbad, CA, USA) to cDNAs using $M-M L V$ reverse transcriptase (Invitrogen, Carlsbad, CA, USA). The cDNAs were used for PCR amplification and gel purification. The purified cDNAs were sequenced with Illumina Hiseq2500 (LC-BIO, Hangzhou, China).
Identification of conserved and novel miRNAs: After sequencing, raw reads were further analyzed using the ACGT101-miR program (LC Sciences, Houston, TX, USA). After removing junk reads and sequences $<18 \mathrm{nt}$ and $>25 \mathrm{nt}$, remaining sequences were aligned using $R$ fam (http://rfam.janelia.org), Cucumber Genome Network database (http://www.icugi.org/cgi-bin/ICuGI/index.cgi) and repeat database (http://www.girinst.org/repbase). The sequences that matched Rfam, mRNA, rRNA, tRNA, snoRNA, snRNA, other non-coding RNAs and repeat sequences were filtered out. The valid sequences were blasted against the reported pre-miRNAs and mature miRNAs in plants from miRBase 21.0 (ftp://mirbase.org/ pub/mirbase/21/). A maximum of one mismatch between target and known miRNAs from miRBase database was allowed. The prediction of novel miRNA was carried out as Cao et al. (2014). UNAfold software were used to predicted hairpin RNA structures (http://mfold.rna.albany. edu/?q=mfold/RNA-Folding-Form). Only miRNAs with stable hairpin structures were considered. miRNAs that are conserved among plants as compared with miRBase and with stable hairpin structures were classified as conserved miRNAs. Novel miRNAs were only considered when minimal folding free energy index (MFEI) of their pre-miRNAs was $\geq 0.80$ in this study.

Data normalization and differential expression analysis: Data normalization was performed according to Cer et al. (2014) with minor modification: 1) Looking for a set of common sequences among all samples to construct a reference data set, in which each data is the copy number median value of a corresponding common sequence. 2) Calculation of the 2-based logarithm with copy numbers of all samples and reference data set $\log _{2}$ (copy\#). 3) Calculation of the difference $\Delta \log _{2}$ (copy\#) between each sample and the reference data set. 4) Selection of sequences $\mid \Delta \log _{2}($ copy\#) $\mid<2$, meaning that less than $\left(2^{2}=\right) 4$ fold change from the reference set. 5) Derivation of linear equations $y=a_{i} x+b_{i}$ from linear regressions between individual samples and the reference set on the subset of selected sequences, where $x$ is $\log _{2}$ (copy\#) of the reference set, $a_{i}$ and $b_{i}$ are the slop and interception, respectively, and $y$ is the expected $\log _{2}$ (copy\#) of library $i$ on a corresponding sequence. 6) Calculation of the mid value $x_{\text {mid }}=[\max (x)-\min (x)] / 2$ of the reference set. Calculation the expected $\log _{2}$ (copy\#) of sample $i y_{i, \text { mid }}=$ $a_{i} x_{\text {mid }}+b_{i}$, make $y_{r \text {,mid }}=x_{\text {mid }}$, and make $\Delta y_{i}=y_{r, \text { mid }}-y_{i, \text { mid }}$, which is the logarithmic correction factor of sample $i$. 7) Derivation of the arithmetic correction factor $f_{i}=2^{\Delta y i}$ of sample $i ; 8$ ) Multiplication of a corresponding arithmetic correction factor to original copy numbers to get corrected copy numbers of individual samples. MicroRNAs were regarded as differentially expressed based on normalized data with the $\chi^{2}$-test and the Fisher-exact test.

The qPCR verification: The total RNAs of samples were isolated using Trizol (Invitrogen, Carlsbad, USA) and digested with DNase I (Fermentas, Loughborough, UK) at $25{ }^{\circ} \mathrm{C}$ for $30 \mathrm{~min}$ to remove DNA. The samples that met a standard of quantity and purity were used for qPCR 
validation of nine miRNAs. The qPCR amplifications were performed as described by Cao et al. (2014). The cDNA synthesis was performed using the One Step PrimeScript $\mathbb{B}$ miRNA cDNA synthesis kit (Takara, Dalian, China). The sequences of the primers for the miRNAs are shown in Table 1 Suppl. The RT-at qPCR amplifications were performed using the SYBR Premix Ex TaqTM kit (Takara, Shiga, Japan). U6snRNA was used as the reference gene for RT-qPCR of miRNAs. The reactions were repeated three times, and the expressions were calculated by the $2^{-\Delta \Delta \mathrm{Ct}}$ method. Three biological replicates of each miRNA were adopted for analysis.

Data assessment and analysis: The sequencing data of this study has been submitted to Gene expression omnibus (GEO) under the accession number of GSE79757 at http://www.ncbi.nlm.nih.gov/geo/query/acc. cgi?acc $=$ GSE79757. To compare the expressions of the miRNAs or the target genes between samples, a modified global normalization (TPM, tags per million) were used to correct copy numbers among different samples. $\mathrm{A}^{2}$-test and Fisher exact test were performed after the raw data were normalized from high-throughput sequencing. $\log _{2}$ ratio was used as the threshold to detect differences in miRNA expressions. The miRNAs were considered to be significantly up-regulated or down-regulated when the $P$ values of the $\chi^{2}$-test and Fisher exact test were $\leq 0.05$ and the $\mid \log _{2}$ ratio $\mid$ was $\geq 1$. qPCR data were analyzed by analysis of variance (ANOVA) using SPSS 16.0 (SPSS Inc., Chicago, IL, USA).

\section{Results}

A total of 11980476 (HH_M), 13684701 (CC_M), 13343713 (HHCC_M), 13036332 (HHCC_Y) reads were obtained. After the redundant reads were screened out and removed, the 7074 090, 7247 941, 8102 408, and 7710834 valid reads in the HH_M, CC_M, HHCC_M, and HHCC Y libraries corresponded to 564614,747864 , 429722 , and 1104828 unique reads, respectively. The small RNAs originating in $C$. $\times$ hytivus and $C$. hystrix which did not perfectly match the $C$. sativus genome were excluded from the analyses because the complete $C$. Xhytivus or $C$. hystrix sequence was unavailable. Thus, the analyses were limited to $C$. sativus small RNAs.

The size of small RNAs were not evenly distributed in each library, ranged from 18 to $25 \mathrm{nt}$ (Fig. 1). Among the unique sequences, 21-nt sRNAs represented the greatest proportion of sequences in the HH_M, HHCC_M, and HHCC_Y libraries, while 22-nt sRN̄As represented the greatest proportion of sequences in the CC_M library (Fig. 1A). However, 21-nt miRNAs represented the greatest proportion in all the four libraries (Fig. 1B).

In total, 546 conserved miRNAs from 407 miRNA precursors and 287 novel miRNAs from 270 miRNA precursors were identified (Table 2 Suppl.). Both conserved and novel miRNAs were differentially expressed among the four libraries (Table 3 Suppl. and Table 4 Suppl.). Firstly, the expression of 15 miRNAs was significantly different between HH_M and CC_M library (Fig. 2). Of these miRNAs, the most up-regulated and down-regulated miRNAs in $\mathrm{HH}_{-} \mathrm{M}$ library comparing to the CC M library were PC-132-5p (19.18-fold) and csamiR397-p3 cme (10.91-fold). Moreover, three of the 15 divergently expressed miRNAs also showed significantly different expression between $\mathrm{HHCC}_{\mathrm{M}}$ and $\mathrm{HH} \mathrm{M}$ library, while four miRNAs showed significant difference in expression between $\mathrm{HHCC}_{-} \mathrm{M}$ and $\mathrm{CC}_{-} \mathrm{M}$ library. In addition to that, nine miRNAs only showed significantly different expression between $\mathrm{HHCC} \mathrm{M}$ and $\mathrm{HH} \mathrm{M}$ libraries, and eight miRNAs between $\mathrm{HHCC}_{\mathrm{M}}$ and CC_M libraries. However, only one of these miRNAs, csamiR 156 a mtr, showed consistently changed expression in HHCC_ $\bar{M}$ compared to both $\mathrm{HH}_{-} \mathrm{M}$ and $\mathrm{CC}$ M libraries. Compared to $\mathrm{HHCC}_{-} \mathrm{M}, \mathrm{HHCC}_{-}^{-} \mathrm{Y}$ showed significantly increased expressions of 15 conserved miRNAs and 12

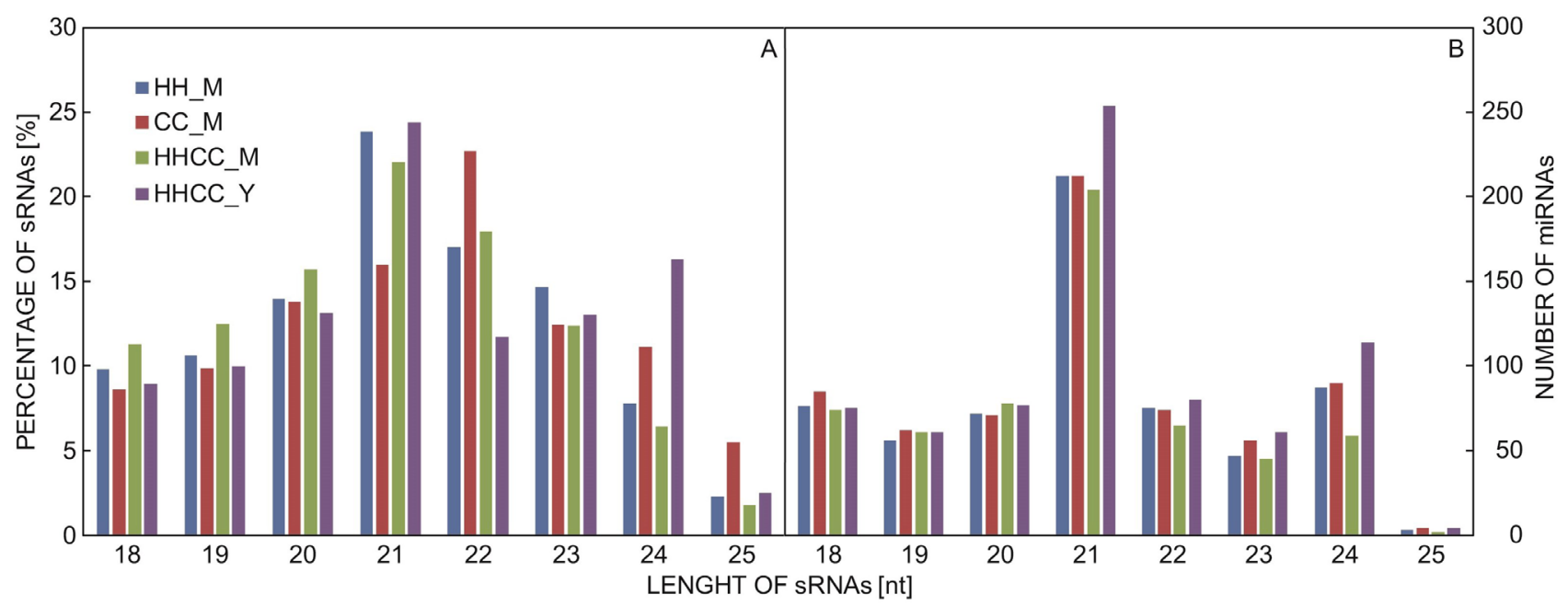

Fig. 1. The length distribution of small RNAs ( $A ; y$-axis refers to the proportion of each length of sRNA in the total number of reads $)$ and miRNAs in the mature leaves of Cucumis hystrix and C. sativus (HH M and CC M), and young and mature leaves of $C . \times$ hytivus $\left(\mathrm{HHCC}_{-} \mathrm{Y}\right.$ and $\left.\mathrm{HHCC} \_\mathrm{M}\right)(B)$. 
novel miRNAs; 11 conserved miRNAs and one novel miRNA showed significantly decreased expression level in HHCC_Y library compared to HHCC_M library. Among these miRNAs, csa-miR171d-p5 cme had the largest increase in expression (34.29-fold), whereas csamiR156b_cme decreased 13.81-fold.

To verify the data obtained from the high-throughput sequencing, qPCR was performed to validate the expression patterns of seven conserved and two novel miRNAs in the samples from four leaves. All the nine miRNAs were detected through qPCR, though some of the miRNAs were detected in relatively low read numbers with deep sequencing. As shown in Fig. 3, the results from qPCR analyses illustrated similar expressions as those detected by next-generation sequencing, suggesting the reliability of our sequencing results.

\section{Discussion}

The success of allopolyploidization between $C$. sativus and a closely related Cucumis species, making it a useful model system to investigate the process of allopolyploidization, as indicated in our previous studies (Chen et al. 2008, 2009). High-throughput sequencing has opened the door for miRNA research, it has permitted research of allopolyploidy effects on miRNAs in Arabidopsis (Ha et al. 2009), wheat (Kenan-Eichler et al. 2011), cotton (Yang et al. 2013, Wang and Zhang 2015) and Brassica species (Ghani et al. 2014, Shen et al. 2014). However, it was still unclear that how the miRNAs were affected by allopolyploidy and the regulation of targeted genes mediated by miRNAs across different species. Moreover, it was unknown whether miRNAs

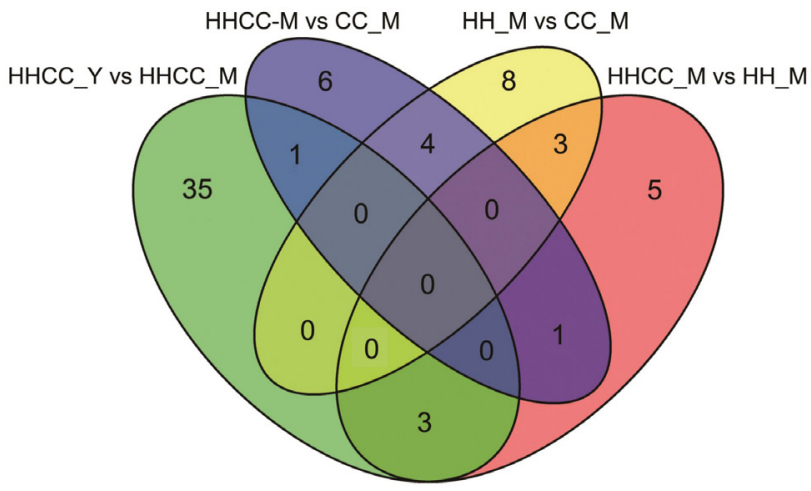

Fig. 2. Venn diagrams of differentially expressed miRNAs in the allotetraploid and its parents in Cucumis $(P<0.05)$.
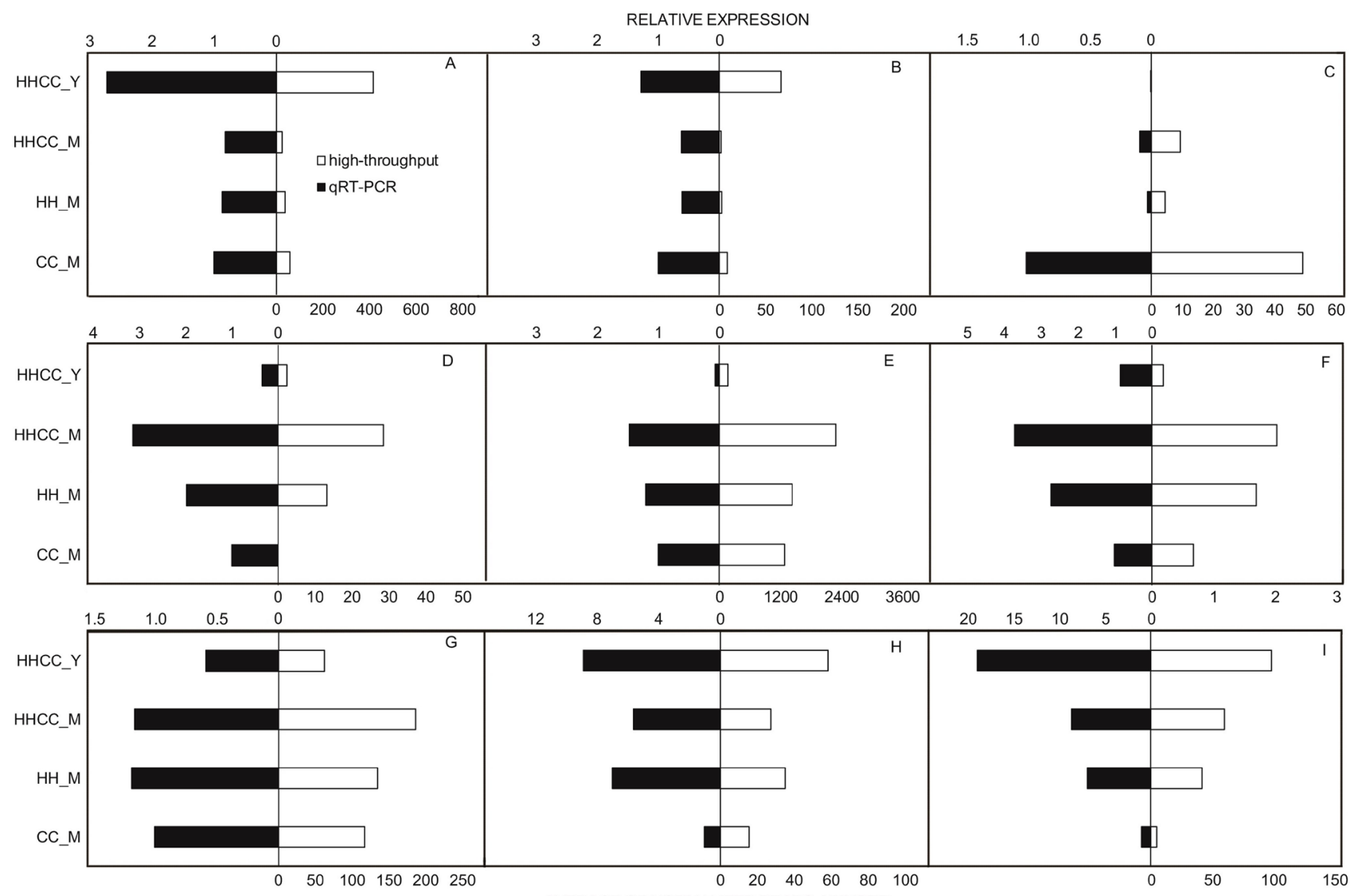

Fig. 3. A comparison of quantitative PCR analyses (black bars) and high-throughput sequencing (white bars) results of miRNAs in Cucumis. $A$ - csa-miR171e-p5_cme; $B$ - csa-miR171d-p5_cme; $C$ - csa-miR397-p3_cme; $D$ - csa-miR171u-p5_gma; $E$ - csa-miR156b_ cme; $F$ - csa-miR156g_ath; $G$ - csa-miR156a_cme; $H$ - PC-260-3p; I - PC-131-3p. 
have contributed to the variation of phenotypes in the process of allopolyploidization, including in the genus of Cucumis. Therefore, we globally investigated the miRNAs expression profile of the synthesized allotetraploid and its parents in Cucumis.

In total, 546 conserved miRNAs and 287 novel miRNAs were identified in the present study, enriched the numbers of Cucumis miRNAs. High abundance of 20 - 24 nt sequences was found, which accounted for $79,78,75$, and $83 \%$ of the clean reads in HH_M, CC_M, HHCC_M, and HHCC_Y libraries, respectively, representing the typical length of plant mature miRNAs (Ng et al. 2012). Of the sRNAs identified in our study, the 24-nt sRNAs showed the highest abundance (Fig. 2), in agreement with the results from rice (Morin et al. 2008), cotton (Yang et al. 2013) and cucumber (Mao et al. 2012). Of the identified miRNAs, 21-nt miRNAs showed the highest proportion, which correlated well to other plant species (Yang et al. 2013).

Allopolyploids obtained by chromosome doubling after hybridisation between species or genera are genetically stable, meaning permanent fixation of heterozygosity $(\mathrm{Ng}$ et al. 2012). MiRNAs play roles in the regulation of a broad range of biological and metabolic processes in the growth and development of plants (Yang et al. 2013, Wang and Zhang 2015). Many miRNAs and their targets are conserved among plants, whereas some are specific to a few plant species. Despite the extensive small RNAs studies in the context of growth and development as well as their tolerance to stress conditions, researches on allopolyploids have been few. Small RNAs were suggested act as a buffer against genomic shock in Arabidopsis allopolyploids, of which the expression variation of miRNAs was suggested to cause changes in gene expression, growth vigour, and adaptation (Ha et al. 2009). Results in hexaploid wheat suggested that miRNA-mediated homoeolog regulation may contribute to heterosis ( $\mathrm{Li}$ et al. 2014). In our data, only 15 out of 833 miRNAs showed expression divergence between the parents, $C$. sativus and $C$. hystrix, which is much less than the results from Brassica hexaploids (Shen et al. 2014) and Arabidopsis allotetraploids (Ha et al. 2009). Nevertheless, it should be noted that these results may consequently biased since the small RNAs originating in $C$. $\times$ hytivus and $C$. hystrix but not perfect matching the $C$. sativus genome were excluded from analysis. Reanalysis after the availability of $C$. ×hytivus and $C$. hystrix genome in future could identify $C . \times$ hytivus and $C$. hystrix specific miRNAs and cement results in the present study.

In polyploids, progenitor-biased gene expression has been widely reported (Wang et al. 2006, Xu et al. 2012). Moreover, progenitor-biased miRNA expression consistent with the progenitor-biased mRNA expression in the allopolyploids has also been reported (Ha et al. 2009, Shen et al. 2014). In our work, one conserved and two novel miRNAs of the 15 divergent miRNAs between the parents showed paternal biased expression in $C$. $\times$ hytivus, whereas two conserved and two novel miRNAs showed maternal biased expression (Fig. 2). In addition, four conserved and four novel miRNAs were divergently expressed between the parents, however, they did not show significantly different expression in $C$. ×hytivus compared with both parents. In other words, the eight conserved and novel miRNAs displayed intermediate expression in the allotetraploid. Moreover, allopolyploidy also gives rise to the activation of new miRNAs and depression of miRNAs. Ten conserved and six novel miRNAs were observed divergently expressed in $C$. ×hytivus in comparison to its parents. Compared with the corresponding diploids, enhanced stress responses of polyploids have been widely observed (Manzaneda et al. 2012). As a result, these nonadditively accumulated miRNAs lead to non-additive expression of their targets in allopolyploids, which may finally lead to non-additive or novel phenotypes in the allopolyploids (Wang et al. 2006, Ha et al. 2009). The varied expression modes of miRNAs suggest more regulation possibilities in synthesized allopolyploids, which therefore may be the advantageous for the increasing of potential for fitness and selective adaptation during the new species establishment.

Conserved miRNAs among different species, however, do not necessarily express the same levels or patterns at different stages within a species (Ha et al. 2008). Ha et al. (2009) reported differentially expressed miRNAs in different organs. It was proposed that every metazoan cell type at each developmental stage could have a distinct miRNA expression profile (Bartel 2004). Our data suggest that it might also apply to plants. The miRNAs have been confirmed for regulating the plant leaf morphogenesis (Palatnik et al. 2003). Moreover, miRNAs have essential roles in coordinating the leaf developmental processes both temporally and spatially (Pulido and Laufs 2010). It was reported that the expressions of miR156 showed dramatic difference between young and old leaves from a single plant in rice (Xie et al. 2012). Consistently, the young and mature leaves of $C$. $\times$ hytivus displayed large difference in miRNAs expression profiles, including 26 conserved and 13 novel miRNAs. Many of these are known to be involved in the leaf growth regulation, such as miR156, miR159, miR166, and miR396 (Pulido and Laufs 2010). Of these miRNAs, three miRNAs from miR156 family, csamiR156a-cme, csa-miR156b-cme, and csa-miR156g-ath, showed increased expression from young to mature leaves, which is consistent with observations in rice (Xie et al. 2012). In addition, 5 miRNAs from miR171 family, csamiR171b-p5-cme, csa-miR171d-p5-cme, csa-miR171ep5-cme, csa-miR171e-3p-mtr, and csa-miR171u-p5-gma, were differently expressed between young and mature leaves of $C$. $\times$ hytivus. miR171 was reported to be crucial for regulating chlorophyll biosynthesis through the gene encoding protochlorophyllide oxidoreductase (POR) (Ma et al. 2014). Recently we have identified chlorophyll deficiency and repression of chlorophyll biosynthesis genes, including the POR gene, in C. ×hytivus (Yu et al. 2018). The results presented here prompts us to further investigate the mechanism of how miRNAs regulate the chlorophyll biosynthesis in the allopolyploids. 


\section{References}

Bartel, D.P.: MicroRNAs: genomics, biogenesis, mechanism, and function. - Cell 116: 281-297, 2004.

Cao, X., Wu, Z., Jiang, F., Zhou, R., Yang, Z.: Identification of chilling stress-responsive tomato microRNAs and their target genes by high-throughput sequencing and degradome analysis. - BMC Genomics 15: 1130, 2014.

Cer, R.Z., Herrera-Galeano, J.E., Anderson, J.J., Bishop-Lilly, K.A., Mokashi, V.P.: miRNA temporal analyzer (mirnaTA): a bioinformatics tool for identifying differentially expressed microRNAs in temporal studies using normal quantile transformation. - Gigascience 3: 20, 2014.

Chen, J., Kirkbride, J.H.: A new synthetic species of Cucumis (Cucurbitaceae) from interspecific hybridization and chromosome doubling. - Brittonia 52: 315-319, 2000.

Chen, J., Staub, J., Adelberg, J., Lewis, S., Kunkle, B.: Synthesis and preliminary characterization of a new species (amphidiploid) in Cucumis. - Euphytica 123: 315-322, 2002.

Chen. J., Staub, J., Qian, C., Jiang, J., Luo, X., Zhuang, F.: Reproduction and cytogenetic characterization of interspecific hybrids derived from Cucumis hystrix Chakr. $\times$ Cucumis sativus L. - Theor. appl. Genet. 106: 688-695, 2003.

Chen, L., Chen, J.: Changes of cytosine methylation induced by wide hybridization and allopolyploidy in Cucumis. - Genome 51: 789-799, 2008.

Chen, L., Lou, Q., Zhuang, Y., Chen, J., Zhang, X., Wolukau, J.N.: Cytological diploidization and rapid genome changes of the newly synthesized allotetraploids Cucumis $\times$ hytivus. Planta 225: 603-614, 2007.

Ghani, M.A., Li, J., Rao, L., Raza, M.A., Cao, L., Yu, N., Zou, X., Chen, L.: The role of small RNAs in wide hybridisation and allopolyploidisation between Brassica rapa and Brassica nigra. - BMC Plant Biol. 14: 272, 2014.

Guan, X., Song, Q., Chen, Z.J.: Polyploidy and small RNA regulation of cotton fiber development. -Trends Plant Sci. 19: 516-528, 2014.

Ha, M., Lu, J., Tian, L., Ramachandran, V., Kasschau, K.D., Chapman, E.J., Carrington, J.C., Chen, X., Wang, X.J., Chen, Z.J.: Small RNAs serve as a genetic buffer against genomic shock in Arabidopsis interspecific hybrids and allopolyploids. - Proc. nat. Acad. Sci. USA 106: 17835-17840, 2009.

Ha, M., Pang, M., Agarwal, V., Chen, Z.J.: Interspecies regulation of microRNAs and their targets. - Biochim. biophys. Acta 1779: 735-742, 2008.

Jiao, Y., Wickett, N.J., Ayyampalayam, S., Chanderbali, A.S., Landherr, L., Ralph, P.E., Tomsho, L.P., Hu, Y., Liang, H., Soltis, P.S., Soltis, D.E., Clifton, S.W., Schlarbaum, S.E., Schuster, S.C., Ma, H., Leebens-Mack, J., De Pamphilis, C.W.: Ancestral polyploidy in seed plants and angiosperms. - Nature 473: 97-100, 2011.

Kenan-Eichler, M., Leshkowitz, D., Tal, L., Noor, E., MelamedBessudo, C., Feldman, M., Levy, A.A.: Wheat hybridization and polyploidization results in deregulation of small RNAs. - Genetics 188: 263-272, 2011.

Li, A., Liu, D., Wu, J., Zhao, X., Hao, M., Geng, S., Yan, J., Jiang, X., Zhang, L., Wu, J., Yin, L., Zhang, R., Wu, L., Zheng, Y., Mao, L.: mRNA and small RNA transcriptomes reveal insights into dynamic homoeolog regulation of allopolyploid heterosis in nascent hexaploid wheat. - Plant Cell 26: 18781900, 2014.
Manzaneda, A.J., Rey, P.J., Bastida, J.M., Weiss-Lehman, C., Raskin, E., Mitchell-Olds, T.: Environmental aridity is associated with cytotype segregation and polyploidy occurrence in Brachypodium distachyon (Poaceae). - New Phytol. 193: 797-805, 2012.

Ma, Z., Hu, X., Cai, W., Huang, W., Zhou, X., Luo, Q., Yang, H., Wang, J., Huang, J.: Arabidopsis miR171-targeted scarecrowlike proteins bind to GT cis-elements and mediate gibberellinregulated chlorophyll biosynthesis under light conditions. PLoS Genet. 10: e1004519, 2014.

Mao, W., Li, Z., Xia, X., Li, Y., Yu, J.: A combined approach of high-throughput sequencing and degradome analysis reveals tissue specific expression of microRNAs and their targets in cucumber. - PloS ONE 7: e33040, 2012.

Morin, R.D., Aksay, G., Dolgosheina, E., Ebhardt, H.A., Magrini, V., Mardis, E.R., Sahinalp, S.C., Unrau, P.J.: Comparative analysis of the small RNA transcriptomes of Pinus contorta and Oryza sativa - Genome Res. 18: 571-584, 2008.

Ng, D.W., Lu, J., Chen, Z.J.: Big roles for small RNAs in polyploidy, hybrid vigor, and hybrid incompatibility. - Curr. Opin. Plant. Biol. 15: 154-161, 2012.

Palatnik, J.F., Allen, E., Wu, X., Schommer, C., Schwab, R., Carrington, J.C., Weigel, D.: Control of leaf morphogenesis by microRNAs. - Nature 425: 257-263, 2003.

Pulido, A., Laufs, P.: Coordination of developmental processes by small RNAs during leaf development. - J. exp. Bot. 61: 1277-1291, 2010.

Shen, Y., Zhao, Q., Zou, J., Wang, W., Gao, Y., Meng, J., Wang, J.: Characterization and expression patterns of small RNAs in synthesized Brassica hexaploids. - Plant mol. Biol. 85: 287 299, 2014.

Wang, J., Tian, L., Lee, H.S., Wei, N.E., Jiang, H., Watson, B., Madlung, A., Osborn, T.C., Doerge, R.W., Comai, L., Chen, Z.J.: Genomewide nonadditive gene regulation in Arabidopsis allotetraploids. - Genetics 172: 507-517, 2006.

Wang, H., Qi, X., Gao, R., Wang, J., Dong, B., Jiang, J., Chen, S., Guan, Z., Fang, W., Liao, Y., Chen, F.: Microsatellite polymorphism among Chrysanthemum sp. polyploids: the influence of whole genome duplication. - Sci. Rep. 4: 6730, 2014.

Wang, Q., Zhang, B.: MicroRNAs in cotton: an open world needs more exploration. - Planta 241: 1303-1312, 2015.

Xie, K., Shen, J., Hou, X., Yao, J., Li, X., Xiao, J., Xiong, L.: Gradual increase of miR156 regulates temporal expression changes of numerous genes during leaf development in rice. Plant Physiol. 158: 1382-1394, 2012.

Xu, Y., Xu, H., Wu, X., Fang, X., Wang, J.: Genetic changes following hybridization and genome doubling in synthetic Brassica napus. - Biochem. Genet. 50: 616-624, 2012.

Yang, X., Wang, L., Yuan, D., Lindsey, K., Zhang, X.: Small RNA and degradome sequencing reveal complex miRNA regulation during cotton somatic embryogenesis. - J. exp. Bot. 64: 1521-1536, 2013.

Yu, X., Wang, X., Hyldgaard, B., Zhu, Z., Zhou, R., Kjaer, K.H., Ouzounis, T., Lou, Q., Li, J., Cai, Q., Rosenqvist, E., Ottosen, C.O., Chen, J.: Allopolyploidization in Cucumis contributes to delayed leaf maturation with repression of redundant homoeologous genes. - Plant J. 94: 393-404, 2018.

Zhang, B., Pan, X., Cannon, C.H., Cobb, G.P.: Anderson TA Conservation and divergence of plant microRNA genes. Plant J. 46: 243-259, 2006. 